\title{
LINEARIZED SUPERSONIC FLOWS WITH AXIAL SYMMETRY*
}

\author{
BY \\ IVALLACE D. HAYES** \\ California Institute of Technology
}

1. Introduction. The study of spatial linearized supersonic flow may be aided by the study of some simple fundamental flows with axial symmetry. Through the principle of superposition, these flows may be combined to give more general flows about various objects and about lifting systems. It is the purpose of this paper to express the equations of linearized supersonic flow in a system of conical coordinates, to develop a theory for fundamental flows with axial symmetry, and to describe examples of such flows and of their combination by superposition.

Various examples of the fundamental equations and solutions here described will be given in later papers, together with the development of some concepts useful in this field.

2. The velocity potential. Steady-state compressible irrotational flow can be described by a velocity potential $\phi$ whose gradient is the velocity vector. Under the assumption that the velocity deviations from a uniform supersonic flow of the Mach number $M$ are small, the differential equation for this potential takes the linear form $^{1,2}$

$$
\phi_{r r}+\frac{1}{r} \phi_{r}+\frac{1}{r^{2}} \phi_{\theta 0}-\left(M^{2}-1\right) \phi_{z z}=0
$$

in cylindrical coordinates.

The fundamental uniform flow is given by the potential $\phi_{0}=V z$ where $V$ is the velocity corresponding to the Mach number $M$. Equation (1) will be considered as yielding velocity deviations which must be added to the velocity of the fundamental flow to describe the net flow.

A new coordinate is introduced to replace the coordinate $r$ :

$$
t=(r / z) \sqrt{M^{2}-1} \text {. }
$$

This quantity is the ratio of the tangents of the polar angle and of the Mach angle. Equation (1) with $r$ eliminated and $t$ introduced becomes

$$
\left(1-t^{2}\right) \phi_{t t}+\frac{1}{t}\left(1-2 t^{2}\right) \phi_{t}+\frac{1}{t^{2}} \phi_{\theta \theta}+2 t z \phi_{t z}-z^{2} \phi_{z z}=0 .
$$

By separation of variables a solution of the form

* Received March 25, 1946.

** This paper was prepared while the writer was employed by the Lockheed Aircraft Corporation.

${ }^{1} \mathrm{R}$. Sauer, Theoretische Einfilhrung in die Gasdynamik, Springer, Berlin, 1943. Reprinted by Edwards Bros., Ann Arbor, 1945.

${ }^{2}$ G. I. Taylor, and J. W. Maccoll, The mechanics of compressible fuids, in Durand, Aerodynamir. theory, vol. 3, Berlin, 1935. 


$$
\phi=z^{n} \Phi(t, \theta)
$$

or

$$
\phi=z^{n} \sin (m \theta+\beta) T(t)
$$

is found. The function $\Phi$ satisfies the equation

$$
\left(1-t^{2}\right) \Phi_{t \imath}+\frac{1}{t}\left(1+2(n-1) t^{2}\right) \Phi_{t}-n(n-1) \Phi+\frac{1}{t^{2}} \Phi_{\theta \theta}=0 .
$$

and may be called the velocity potential for generalized conical flow. If $n=1$, the function $\Phi$ describes conical flow. The function $T$ satisfies the equation

$$
\left(1-t^{2}\right) T_{\imath}+\frac{1}{t}\left(1+2(n-1) t^{2}\right) T_{\imath}-\frac{1}{t^{2}}\left(m^{2}+n(n-1) t^{2}\right) T=0 .
$$

Superposition of solutions of the type of (4a), (4b) will give a general solution.

The velocity components are

$$
u=\frac{\sqrt{M^{2}-1}}{z} \phi_{\iota}
$$

in the radial direction,

$$
v=\frac{\sqrt{M^{2}-1}}{t z} \phi_{\theta}
$$

in the azimuthal direction, and

$$
w=\phi_{z}-\frac{t}{z} \phi_{t}
$$

in the axial direction. The pressure in linearized supersonic flow is given in terms of the velocity components by

$$
p=-\rho\left(V w+\frac{u^{2}+v^{2}}{2}\right)
$$

and the pressure coefficient by

$$
C_{p}=-2\left(\frac{w}{V}+\frac{u^{2}+v^{2}}{2 V^{2}}\right) .
$$

The part of Eqs. (8a), (8b) in $u$ and $v$ is not necessary if $w$ is of the same magnitude as $u$ and $v$. In many important cases, however, $u^{2}+v^{2}$ is of the same magnitude as $V w$ and Eqs. (8a), (8b) must be used in its complete form. In these cases the validity of the solution should be checked.

The singularity of (5) or (6) at $t= \pm 1$ corresponds to the two Mach cones extending from the origin in the three dimensional flow. Various ranges of $t$ correspond to various regions of flow, as shown in the following table. 


\begin{tabular}{|c|c|}
\hline Range of $t$ & Region of Flow \\
\hline $0 \leqq t<1$ & inside downstreain cone \\
\hline$-1<t \leqq 0$ & inside upstream cone \\
\hline $\left.\begin{array}{c}1<t<\infty \\
-\infty<t<-1\end{array}\right\}$ & outside both cones \\
\hline
\end{tabular}

3. Solutions of the differential equation (6). The parameter $n$ is restricted to integers and the parameter $m$ to non-negative integers. The differential equation for $T$, Eq. (6), has regular singularities of exponents $(+m,-m)$ at $t=0,\left(0, n+\frac{1}{2}\right)$ at $t= \pm 1$, and $(-n, 1-n)$ at $t=\infty$. The solutions about the origin are

$$
\begin{aligned}
T & =t^{m} F\left(\frac{-n+m}{2}, \frac{-n+m+1}{2} ; 1+m ; t^{2}\right) \\
& =t^{m}\left(1-t^{2}\right)^{n+\frac{1}{2}} F\left(\frac{n+m+1}{2}, \frac{n+m+2}{2} ; 1+m ; t^{2}\right), \\
T & =t^{-m} F\left(\frac{-n-m}{2}, \frac{-n-m+1}{2} ; 1-m ; t^{2}\right) \\
& =t^{-m}\left(1-t^{2}\right)^{n+1} F\left(\frac{n-m+1}{2}, \frac{n-m+2}{2} ; 1-m ; t^{2}\right),
\end{aligned}
$$

where $F$ denotes the hypergeometric function. The solution of negative exponent, Eqs. (10a), (10b) is not well defined.

It is of considerably more value to express the solutions about $t^{2}=1$, since then both solutions are well defined and two distinct types of solution may be distinguished. One type of solution, designated as type $I$, is the solution of zero exponent at $t^{2}=1$ and is real throughout the range of $t$. The resulting solution for $\phi$ has no singularity on the Mach cones. The other type of solution, designated as type II, is the solution of exponent $n+\frac{1}{2}$ at $t^{2}=1$ and is real only for $t^{2}<1$ or only for $t^{2}>1$. The resulting solution for $\phi$ is defined only within the Mach cones or only outside the Mach cones. These solutions may be expressed as follows:

I)

$$
\begin{aligned}
T & =t^{m} F\left(\frac{-n+m}{2}, \frac{-n+m+1}{2} ;-n+\frac{1}{2} ; 1-t^{2}\right) \\
& =t^{-m} F\left(\frac{-n-m}{2}, \frac{-n-m+1}{2} ;-n+\frac{1}{2} ; 1-t^{2}\right),
\end{aligned}
$$

II)

$$
\begin{aligned}
T & =t^{m}\left(1-t^{2}\right)^{n+\frac{1}{3}} F\left(\frac{n+m+1}{2}, \frac{n+m+2}{2} ; n+\frac{3}{2} ; 1-t^{2}\right) \\
& =t^{-m}\left(1-t^{2}\right)^{n+\frac{1}{2} F}\left(\frac{n-m+1}{2}, \frac{n-m+2}{2} ; n+\frac{3}{2}: 1-t^{2}\right) .
\end{aligned}
$$


Three special cases are distinguished according to the relative values of $n$ and $m$ :

case A: $-\infty<n \leqq-m-1$,

case B: $-m \leqq n \leqq m-1$,

case $\mathrm{C}: m \leqq n<\infty$

The distribution of these cases for small values of $m$ and $n$ is shown in the table:

\begin{tabular}{c|cccccc}
\hline$n$ & -3 & -2 & -1 & 0 & 1 & 2 \\
\hline 0 & $A$ & $A$ & $A$ & $C$ & $C$ & $C$ \\
1 & $A$ & $A$ & $B$ & $B$ & $C$ & $C$ \\
2 & $A$ & $B$ & $B$ & $B$ & $B$ & $C$ \\
3 & $B$ & $B$ & $B$ & $B$ & $B$ & $B$ \\
\hline
\end{tabular}

From a consideration of Eqs. (9) to (12) the forms of the two types of solutions in the various cases may be found. For all solutions except solutions I-A (i.e., solutions of type $\mathrm{I}$ in case A) and solutions II-C, the form is explicit in terms of a polynomial in $t^{2}$ or in $\left(1-t^{2}\right)$. Solutions I-A and II-C have logarithmic singularities at $t=0$ and are discussed later. The polynomial forms are expressed as follows:

\begin{tabular}{|c|c|c|c|}
\hline Solutions & Form & $\begin{array}{c}\text { Order of } P\left(t^{2}\right) \\
\text { whichever is integral of }\end{array}$ & $\begin{array}{l}\text { Equation for } \\
\text { Calculation }\end{array}$ \\
\hline $\begin{array}{r}\text { I-A } \\
\text { II-A } \\
I-B \\
\text { II-B } \\
I-C \\
\text { II-C }\end{array}$ & $\begin{array}{l}\quad \text { logarithmic } \\
t^{m}\left(1-t^{2}\right)^{n+1} P\left(t^{2}\right) \\
t^{-m} P\left(t^{2}\right) \\
t^{-m}\left(1-t^{2}\right)^{n+1} P\left(t^{2}\right) \\
t^{m} P\left(t^{2}\right) \\
\quad \text { logarithmic }\end{array}$ & $\begin{array}{l}\frac{1}{\frac{1}{2}(-n-m-1) \text { or } \frac{1}{2}(-n-m-2)} \\
\frac{1}{2}(n+m) \text { or } \frac{1}{2}(n+m-1) \\
\frac{1}{2}(-n+m-1) \text { or } \frac{1}{2}(-n+m-2) \\
\frac{1}{2}(n-m) \text { or } \frac{1}{2}(n-m-1)\end{array}$ & $\begin{array}{r}(9 b) \text { or }(12 a) \\
(10 a) \text { or }(11 b) \\
(10 b) \text { or }(12 b) \\
(9 a) \text { or }(11 a) \\
\end{array}$ \\
\hline
\end{tabular}

There is a connection between the solutions of Eq. (6) and the Legendre functions with the same values of $n$ and $m$, except that $-n-1$ is used when $n$ is negative. However, since Legendre functions are customarily defined only for $m \leqq n$ or $m \leqq n-1$, respectively, they are of assistance here only in cases $\mathrm{A}$ and $\mathrm{C}$. The polynomial solutions are

$$
\begin{aligned}
& T=\left(1-t^{2}\right)^{n / 2} P_{n}^{m}\left[\left(1-t^{2}\right)^{-\frac{1}{2}}\right], \\
& T=\left(1-t^{2}\right)^{n / 2} P_{-n-1}^{m}\left[\left(1-t^{2}\right)^{-\frac{1}{3}}\right] .
\end{aligned}
$$

These solutions are most easily obtained by transforming Eq. (1) into Laplace's equation by introducing the variable $i z / \sqrt{M^{2}-1}$.

The polynomial solutions may be obtained in another form from an expression given by A. R. Forsyth, ${ }^{3}$ and the law for differentiating the hypergeometric functions. When $n$ is not positive, these solutions are

${ }^{3}$ A. R. Forsyth, $A$ treatise on differential equations, 6th ed., Macmillan, London, 1933, p. 235. 
1)

$$
\begin{aligned}
& T=t^{-m} \frac{d^{-n}}{d\left(t^{2}\right)^{-n}}\left[\left(1+\sqrt{1-t^{2}}\right)^{-n+m}+\left(1-\sqrt{1-t^{2}}\right)^{\cdots+n+m}\right\rfloor, \\
& T=t^{ \pm m} \frac{d^{-n}}{d\left(t^{2}\right)^{-n}}\left[\left(1+\sqrt{1-t^{2}}\right)^{-n \mp m}-\left(1-\sqrt{1-t^{2}}\right)^{-n \mp m}\right\rfloor,
\end{aligned}
$$

and when $n+1$ is not negative,

I) $T=t^{ \pm m}\left(1-t^{2}\right)^{n+\frac{1}{2}} \frac{d^{n+1}}{d\left(t^{2}\right)^{n+1}}\left[\left(1+\sqrt{1-t^{2}}\right)^{n+1 \mp m}-\left(1-\sqrt{1-t^{2}}\right)^{n+1 \mp m}\right]$,

II) $T=t^{-m}\left(1-t^{2}\right)^{n+1} \frac{d^{n+1}}{d\left(t^{2}\right)^{n+1}}\left[\left(1+\sqrt{1-t^{2}}\right)^{n+1+m}+\left(1-\sqrt{1-t^{2}}\right)^{n+1+m}\right]$.

4. Logarithmic solutions. The logarithmic solutions I-A and II-C are most easily expressed in terms of the Legendre functions, as in Eqs. (13a), (13b). They are

$$
\begin{aligned}
& T=\left(1-t^{2}\right)^{n / 2} Q_{-n-1}^{m}\left[(1-t)^{-1}\right], \\
& T=\left(1-t^{2}\right)^{n / 2} Q_{n}^{m}\left[\left(1-t^{2}\right)^{-j}\right] .
\end{aligned}
$$

Since the validity of Eqs. (14) to (17) does not depend upon $m$ being an integer, and since an appropriate solution of the form of these equations vanishes as a $\log$ arithmic solution is approached, the logarithmic solutions may also be obtained by differentiating such solutions with respect to $m$. The logarithmic solutions in this form are

$$
\begin{aligned}
& \text { I-A) } \quad T=t^{ \pm m} \frac{d^{-n}}{d\left(t^{2}\right)^{-n}}\left[\left(1+\sqrt{1-t^{2}}\right)^{-n \mp m} \log \left(1+\sqrt{1-t^{2}}\right)\right. \\
& \left.+\left(1-\sqrt{1-t^{2}}\right)^{-n \mp m} \log \left(1-\sqrt{1-t^{2}}\right)\right], \\
& \text { II-C) } T=t^{ \pm m}\left(1-t^{2}\right)^{n+\frac{1}{2}} \frac{d^{n+1}}{d\left(t^{2}\right)^{n+1}}\left[\left(1+\sqrt{1-t^{2}}\right)^{n+1 \mp m} \log \left(1+\sqrt{1-t^{2}}\right)\right. \\
& \left.+\left(1-\sqrt{1-t^{2}}\right)^{n+1 \mp m} \log \left(1-\sqrt{1-t^{2}}\right)\right] .
\end{aligned}
$$

5. Generating equations. The fundamental equation, Eq. (1), expressed in Cartesian coordinates is invariant under differentiation with respect to any of these coordinates. Solutions of the type of Eq. (4b) expressed in Cartesian coordinates and differentiated with respect to these coordinates are still solutions of Eqs. (1) and (3). This fact permits a given solution of parameters $n$ and $m$ to yield solutions of parameters $n-1$ and $m, m+1$, or $m-1$ :

$$
\begin{aligned}
T(n-1, m) & =n T-t T_{t}=-t^{n+1} \frac{d}{d t}\left(t^{n} T\right), \\
T(n-1, m+1) & =\frac{m}{t} T-T_{t}{ }^{\prime}=-t^{+m} \frac{d}{d t}\left(t^{-m} T\right), \\
T(n-1, m-1) & =\frac{m}{t} T+T_{t}=+t^{-m} \frac{d}{d t}\left(t^{+m} T\right) .
\end{aligned}
$$

The procedures yielding these new solutions can be considered procedures of super- 
position; for example, one solution superposed on its negative an infinitesimal distance downstream yields the new solution given by Eq. (21a)

These equations are not to be considered recurrence relations, as no system has been established for specifying solutions with respect to the multiplicative arbitrary constant.

In a similar manner solutions with the parameter $n$ increased by 1 may be obtained by reversing Eqs. (21a), (21b), and (21c) with suitable integrations.

6. Integral relation. An integral relation connecting two solutions whose parameters differ in value may be obtained either from the corresponding relation for the Legendre functions or directly from Eq. (6). If $T_{1}$ denotes a solution corresponding to $n_{1}$ and $m_{1}$ and $T_{2}$ a solution corresponding to $n_{2}$ and $m_{2}$, the relation is

$$
\begin{aligned}
& \left(m_{1}^{2}-m_{2}^{2}\right) \int_{a}^{b} t^{-1}\left(1-t^{2}\right)^{-\frac{1}{\left(n_{1}+n_{2}+1\right)}} T_{1} T_{2} d t \\
& +\left(n_{1}-n_{2}\right)\left(n_{1}+n_{2}+1\right) \int_{a}^{b} t\left(1-t^{2}\right)^{-\frac{1}{2}\left(n_{1}+n_{2}+3\right)} T_{1} T_{2} d t \\
& =\left[t\left(1-t^{2}\right)^{-\frac{1}{2}\left(n_{1}+n_{2}-1\right)}\left(T_{2} \frac{d T_{1}}{d t}-T_{1} \frac{d T_{2}}{d t}\right)\right. \\
& \left.+\left(n_{1}-n_{2}\right) t^{2}\left(1-t^{2}\right)^{-1\left(n_{1}+n_{2}+1\right)} T_{1} T_{2}\right]\left.\right|_{a} ^{b} .
\end{aligned}
$$

Setting $n_{1}=n_{2}$ or $m_{1}=m_{2}$, we obtain simpler equations as special cases which may be used to obtain orthogonality relations between solutions.

7. Two-dimensional cross-filow. The solutions of type I for which $m=|n|$ are given by $T=t^{n}$. The corresponding solutions for $\phi$ in cylindrical coordinates are

$$
\phi=r^{n} \sin (|n| \theta+\beta) \text {. }
$$

These solutions give two-dimensional cross-flow because of the fact that $z$ does not appear. This cross-flow, as a result of the linearizing assumptions, appears as an incompressible flow.

8. Conical flow. The solutions of either type for which $n=1$ give solutions of conical flow, of which only those of type II are here treated. Since the exponent of these solutions at $t^{2}=1$ is $3 / 2$, both the potential and the velocity vanish on the Mach cone. The first few solutions are:

$$
\begin{array}{ll}
m=0) & \sqrt{1-t^{2}}-\tanh ^{-1} \sqrt{1-t^{2}}, \\
m=1) & t^{-1} \sqrt{1-t^{2}}-t \tanh ^{-1} \sqrt{1-t^{2}}, \\
m=2) & t^{-2}\left(1-t^{2}\right)^{3 / 2}, \\
m=3) & t^{-3}\left(1-t^{2}\right)^{3 / 2},
\end{array}
$$

The flow about an infinitesimal circular cone at zero incidence is given by the first solution (II, 1, 0), the solution of type II with $n=1$ and $m=0$. The flow about the same cone at a small angle of attack is obtained by superposing solutions (II, 1, 1) and $(I, 1,1)$ with appropriate constants on the (II, 1, 0) solution. A standard treatment of this case will be found on pp. 46 to 49 of Sauer's book. ${ }^{1}$

9. Infinitesimal horseshoe vortices. An infinitesimal horseshoe vortex can be represented by a semi-infinite line dipole in the same manner as a planar vortex 
system can be represented by a planar dipole system. Thus a lifting element and other lifting systems can be represented by solutions of type II with $m=1$, as shown in the following table.

\begin{tabular}{|c|c|c|}
\hline Solution & $\begin{array}{l}\text { Semi-Infinite Line } \\
\text { Dipole of Strength }\end{array}$ & $\begin{array}{l}\text { Designation in Terms of } \\
\text { Lifting Properties }\end{array}$ \\
\hline $\begin{array}{ll}(\text { II },-1,1): & t^{-1}\left(1-t^{2}\right)^{-\frac{1}{2}} \\
\text { (II, } 0,1): & t^{-1}\left(1-t^{2}\right)^{+1} \\
\text { (II, } 1,1): & t^{-1}\left(1-t^{2}\right)^{+\frac{1}{3}}-t \tanh ^{-1}\left(1-t^{2}\right)^{+\frac{1}{2}}\end{array}$ & $\begin{array}{l}\text { Constant } \\
\text { Proportional to } z \\
\text { Proportional to } z^{2}\end{array}$ & $\begin{array}{l}\text { "Lifting element" } \\
\text { "Lifting line" } \\
\text { "Lifting infinitesimal triangle" }\end{array}$ \\
\hline
\end{tabular}

The "lifting element" solution, since the potential has exponent $-\frac{1}{2}$ and the velocities $-\frac{3}{2}$ at $t^{2}=1$, has a troublesome singularity on the Mach cones. A simpler singularity has the "lifting line" solution, whose potential vanishes and whose velocities have exponent $-\frac{1}{2}$ at $t^{2}=1$. When these solutions are superposed to give lifting systems of finite dimension, the singularity in the velocities usually disappears. The third solution is the same as the one which gives the lift on an inclined circular cone. Examples of the superposition of such solutions to form a lifting system will be given in a later paper.

10. The acceleration potential. Since the axial velocity component $w$ is a derivative of the velocity potential in Cartesian coordinates, it satisfies the same equations, Eqs. (1) to (6), as does the velocity potential. The acceleration potential, whose fundamental theory will be found in a paper by $L$. Prandtl, ${ }^{4}$ equals $-p / \rho$ for linearized flow, and also equals $V w+\frac{1}{2}\left(u^{2}+v^{2}\right)$ from Eq. (8a). Hence the approximate acceleration potential defined by $\psi=V w$ satisfies Eqs. (1) to (6). The relation of this quantity to the velocity potential for a given fundamental flows is of the type which leads to Eq. (21a), and hence the corresponding acceleration potential is given by a solution with $n$ decreased by 1 . It is important to note that this does not give the true linearized acceleration potential where $\boldsymbol{u}^{2}+v^{2}$ is not of smaller magnitude than $V w$. Thus the two-dimensional cross-flow described above has no approximate acceleration potential, and the acceleration potential is given incorrectly in the vicinity of the axis for other flows. However, the true acceleration potential may not itself be superposed, and of ten the difference between the approximate and true linearized acceleration potential disappears under superposition.

The "lifting element," "lifting line," and "lifting infinitesimal triangle" have approximate acceleration potential solutions (II, $-2,1),($ II $,-1,1)$, and (II, 0,1$)$, respectively. With conical flow, the approximate acceleration potential is a function only of $t$ and $\theta$ and can be shown to satisfy Laplace's equation in two dimensions.

4. Prandtl, Theorie des Flugzeugtragfilgels in zusammendrï̈ckbarem Medium, Luftfahrtforschung, 13,313 (1936). 